\title{
Analysis of the Results of Treatment of Destructive Pulmonary Tuberculosis and Possibilities of Prediction of its Outcomes
}

\section{Sheifer YA ${ }^{1 *}$ and Gelberg IS ${ }^{2}$}

${ }^{1}$ Senior Lecturer of the Department of Phthisiopulmonology, "Grodno State Medical University", Republic of Belarus

${ }^{2}$ Professor of the Department of Phthisiopulmonology, "Grodno State Medical University", Republic of Belarus

*Correspondingauthor:YuASheifer,Senior Lecturer of the Department of Phthisiopulmonology, "Grodno State Medical University", 230009, Grodno, st. Gorky 80, Republic of Belarus, Tel: 80152434392; 80297820963; Email: jura-med@mail.ru

\section{Abstract}

Purpose of the study: Analyze the results of chemotherapy for destructive forms of pulmonary tuberculosis and develop a method for predicting its outcomes.

The analysis of long-term results of treatment was carried out in 296 patients with destructive pulmonary TB who were inpatient treatment at the health care institution Grodno Regional Clinical Center "Phthisiology". The design included a randomized, controlled, retrospective, prospective cohort study. In patients with persisting destruction of lung tissue, despite the achievement of abacillation by the end of the intensive phase of chemotherapy, a high proportion of ineffective treatment (28.6-57.4\%), transition to fibro-cavernous tuberculosis (16.7-35.3\%) was established, amplification of drug resistance, the emergence of widespread drug resistance of M. tuberculosis (up to 38.2\%), which causes a decrease in the rate of successful treatment (up to 39.7\%) and a high rate of deaths (21.4-33.8\%). A method has been developed for predicting the preservation of decay cavities in patients with destructive pulmonary tuberculosis during chemotherapy, according to which the predictors are the clinical form of pulmonary tuberculosis, the number of cavities, the presence of alcohol dependence syndrome, and the duration of abacillation. Based on the results obtained, a regression equation was constructed to predict the possible preservation of decay cavities against the background of chemotherapy:

$$
\mathrm{Z}=6.456-4.907 * \mathrm{CF}-3.682 * \mathrm{NC}-2.169 * \text { ADS-A, }
$$

where: $\mathrm{CF}$ (clinical form) = 0 for infiltrative pulmonary TB in the decay phase; CF $=1$ with cavernous; NC (number of decay cavities) $=0$ in the presence of 1 decay cavity; $\mathrm{NC}=1$ in the presence of 2 cavities or more; ADS- alcohol dependence syndrome ( 0 - no sign, 1 - there is a sign); $\mathrm{A}=0$ Abacillation received within 3 months; $\mathrm{A}=4.115$ - 3-4 months; $\mathrm{A}=5.189$ - 5-6 months; $A=25.249$ - in the absence of abacillation. The sensitivity of the method is $97.0 \%$, the specificity is $80.6 \%$. The method makes it possible to substantiate a differentiated approach to complex treatment with the timely use of surgical or collapse therapy methods.

Keywords: Destructive Tuberculosis; Prognosis; Predictors; Outcomes; Cohort; Multidrug Resistance of Mycobacterium Tuberculosis; Long-Term Results; Specificity; Sensitivity 
Abbreviations: TB: Tuberculosis; DR: Drug-Resistant; MDR: Multiple Drug Resistance; XDR: extensively drug Resistant; FCN: Fibro cavernous; MG: Main Group; CT: Chemotherapy; ADS: Alcohol Dependence Syndrome; A: Abacillation; CF: Clinical Form; NC: Number of Decay Cavities.

\section{Introduction}

At present, the infectious type of pathology dominates in developing countries [1,2]. Despite the improvement in the epidemiological situation, tuberculosis (TB) poses a significant threat to public health, a disease with profound economic and social consequences. In the modern period, the main problem of phthisiology is the increase in the proportion of drug-resistant TB (DR-TB). In the structure of DR-TB has an important place multiple drug resistance (MDR), extensively drug resistant (XDR) [2].

Modern destructive pulmonary TB in $60-70 \%$ of cases is caused by DR strains of MBT, and $35-40 \%$ of patients have a progressive course [3]. Among all forms of TB lung epidemiologically most dangerous fibrocavernous TB (FCN): being the chronic form, it often leads to non-reversible changes in the body, and is accompanied by copious MDR and XDR MBT $[4,5]$.

"Insufficient effectiveness of treatment of patients with DR-TB is a known fact" [6]. The problem of DR-TB treatment continues to be the most urgent in modern phthisiology for the entire world community, cure rates do not exceed $65 \%$ $[6,7]$.

The achieved results of effective treatment of patients with MDR MBT remain insufficient to improve the epidemic situation. A high proportion of ineffective treatment outcomes creates conditions for the spread of MBT strains with MDR and XDR [6].

According to the WHO, the assessment of treatment outcomes evaluated only the termination of bacteria as epidemiologically this indicator is the most significant. Meanwhile, changes in clinical symptoms, radiological manifestations (healing of decay cavities), as well as restoration of working capacity are extremely important for the patient and reflect the effectiveness of the main course of treatment $[1,8,5]$.

The elimination of the cavity is the main condition for cure in destructive pulmonary $\mathrm{TB}$, especially in the case of resistance of the pathogen [9-11].

\section{The Purpose of the Study}

To analyze the results of chemotherapy for destructive forms of pulmonary tuberculosis and develop a method for predicting its outcomes to optimize the treatment of destructive forms of tuberculosis, mainly with MDR MBT.

\section{Material and Methods}

Conducted a retrospective analysis of treatment results of 296 patient s with destructive pulmonary TB, were hospitalized at "Grodno Regional Clinical Center "Phthisiology". The design included a randomized, controlled, retrospective, prospective cohort study.

Statistical processing of the results was carried out using the Statistica for Windows data processing package, version 10.0 and the Excel office application. Differences were considered statistically significant at $p<0,05$. To build a mathematical forecasting model using the analysis "Logistic regression", the predictive value of the studied indicators was determined. Having received the final set of the most important variables (predictors), a regression equation was constructed. The calculation of sensitivity, specificity was carried out on the basis of the construction and analysis of ROC- curves.

Criteria for inclusion in a retrospective study: the presence of lung tissue destruction in focal, infiltrative, subacute Disseminated pulmonary TB; tuberculomas; Cavernous TB of the lungs.

Exclusion criteria: tuberculous processes without destruction, miliary, chronic disseminated pulmonary TB, fibrous-cavernous pulmonary TB, cirrhotic pulmonary TB, caseous pneumonia.

The main group $(M G)(n=110)$ consisted of persons with destructive pulmonary TB who were discharged from the hospital with preserved destruction of lung tissue. Comparison group $(n=81)$ - persons discharged with the closure of decay cavities. Given the differences in the schemes of chemotherapy (CT), patients were divided into treatment cohorts in 2009 - 2012. $(n=108)$ (order of the Ministry of Health of the Republic of Belarus dated 12.01.2009 No 11) and the cohort of 2012 - 2015. ( $\mathrm{n}=83$ ) (order of the Ministry of Health of the Republic of Belarus dated 22.08.2012 No. 939). Each cohort was divided, respectively, into the main group and the comparison group. Patient characteristics are presented in Table 1. 


\begin{tabular}{|c|c|c|c|c|c|c|c|c|c|c|}
\hline \multirow{4}{*}{ Indicator name } & \multicolumn{4}{|c|}{ By the end of 2009-2012. } & \multirow{4}{*}{$\mathbf{R}$} & \multicolumn{4}{|c|}{ Cohort 2009-2012 gg. } & \multirow{4}{*}{$\mathbf{p}$} \\
\hline & \multirow{2}{*}{\multicolumn{2}{|c|}{$\begin{array}{c}\mathrm{OG}-\mathrm{CV}+ \\
(\mathrm{n}=68)\end{array}$}} & \multirow{2}{*}{\multicolumn{2}{|c|}{$\begin{array}{l}\text { HS - CV- } \\
(n=40)\end{array}$}} & & \multirow{2}{*}{\multicolumn{2}{|c|}{$\begin{array}{c}O G-C V+ \\
(n=42)\end{array}$}} & \multirow{2}{*}{\multicolumn{2}{|c|}{$\begin{array}{l}\text { HS - CV- } \\
(n=41)\end{array}$}} & \\
\hline & & & & & & & & & & \\
\hline & abs. & $\%$ & abs. & $\%$ & & abs. & $\%$ & abs. & $\%$ & \\
\hline \multicolumn{11}{|l|}{ Clinical TB } \\
\hline infiltrative & 57 & 83.8 & 34 & 85 & $>0.05$ & 37 & 88.1 & 37 & 90.3 & $>0.05$ \\
\hline cavernous & 8 & 11.8 & 6 & 15 & $>0.05$ & 2 & 4.8 & 1 & 2.4 & $>0.05$ \\
\hline disseminated & 1 & 1.5 & 0 & 0 & $>0.05$ & 3 & 7.1 & 1 & 2.4 & $>0.05$ \\
\hline tuberculoma & 2 & 2.9 & 0 & 0 & $>0.05$ & 0 & 0 & 2 & 4.9 & $>0.05$ \\
\hline \multicolumn{11}{|l|}{ Number of cavities } \\
\hline 1 cavity & 46 & 67.6 & 32 & 80 & $>0.05$ & 35 & 83.3 & 34 & 82.9 & $>0.05$ \\
\hline 2 cavities or more & 22 & 32.4 & 8 & 20 & $>0.05$ & 7 & 16.7 & 7 & 17.1 & $>0.05$ \\
\hline \multicolumn{11}{|l|}{ Prevalence } \\
\hline limited & 2 & 2.9 & 4 & 15 & $<0.05$ & 8 & 19.1 & 14 & 34.1 & $>0.05$ \\
\hline common & 66 & 97.1 & 36 & 85 & $>0.05$ & 34 & 80.9 & 27 & 65.9 & $<0.05$ \\
\hline \multicolumn{11}{|l|}{ Revealing } \\
\hline first identified & 28 & 41.2 & 22 & 55 & $>0.05$ & 15 & 35,7 & 22 & 53.7 & $>0.05$ \\
\hline re-treated & 40 & 58.8 & 18 & 45 & $>0.05$ & 27 & 64.3 & 19 & 46.3 & $>0.05$ \\
\hline \multicolumn{11}{|l|}{ Aggravating factors } \\
\hline came from prison & 8 & 11.8 & 4 & 10 & $>0.05$ & 6 & 14.2 & 3 & 7.3 & $>0.05$ \\
\hline alcohol addiction & 41 & 60.3 & 25 & 62.5 & $>0.05$ & 22 & 52.4 & 18 & 43.9 & $>0.05$ \\
\hline With diabetes & 4 & 5.9 & 2 & 5 & $>0.05$ & 3 & 7.1 & 5 & 12.2 & $>0.05$ \\
\hline several factors & 23 & 33.8 & 13 & 32.5 & $>0.05$ & 15 & 35,7 & 11 & 26.8 & $>0.05$ \\
\hline
\end{tabular}

Table 1: Patient characteristics.

Note: $p>0.05$ - no significant differences between indicators in pairwise comparison in groups; $\mathrm{p}$ - Fisher expact test "two tailed".

As shown in Table 1, both cohorts are dominated by males with infiltrative pulmonary TB, widespread tuberculosis, and a high proportion of risk factors. The groups were comparable by age and sex.

Of the 191 patients and with the destructive pulmonary TB (retrospective study) were randomly group was formed - 105, to predict the likelihood of conservation decay cavities on the background of chemotherapy evaluated for its performance in the examination sample $(n=105)$. These groups were comparable in terms of compared characteristics.

\section{Research Results}

The duration of inpatient treatment of patients with MDR MBT, cohort 2009 - 2012, in both groups ranged from 3 to 12 months or more.

\begin{tabular}{|c|c|c|c|c|c|}
\hline \multirow{2}{*}{ Outcomes } & \multicolumn{2}{|c|}{ CV + } & \multicolumn{2}{|c|}{ CV- } & \multirow{2}{*}{$\mathbf{p}$} \\
\cline { 2 - 5 } & $\mathbf{n}$ & $\mathbf{\%}$ & $\mathbf{n}$ & $\mathbf{\%}$ & $<0.05$ \\
\hline Cure & 16 & 23.5 & 25 & 62.5 & $<0.05$ \\
\hline Treatment completed & 3 & 4.4 & 6 & 15.0 & $<0.05$ \\
\hline Successful treatment & 19 & 27.9 & 31 & 77.5 & $<0.05$ \\
\hline Ineffective treatment & 39 & 57.4 & 3 & 7.5 & - \\
\hline Death & 0 & 0 & 0 & 0 & \\
\hline a) from tuberculosis & 2 & 2.9 & 1 & 2.5 & $>0.05$ \\
\hline b) from other reasons & 8 & 11.8 & 5 & 12.5 & $>0.05$ \\
\hline
\end{tabular}

Table2: Results of treatment of patients with MDR MBT by 24 months, cohort $2009-2012$.

Note: $\mathrm{p}$ - test $\chi 2$. 
Although there are no significant differences in the length of stay of patients in the hospital, it should be noted that patients discharged with destruction of lung tissue in $17.6 \%$ of cases continued treatment for up to 12 months or more. By the time of discharge from the hospital, the abacillation rate in both groups was $100 \%$. The results of treatment of patients of these groups after 24 months are presented in Table 2.

Thus, the percentage of successful treatment of patients discharged with destruction of lung tissue within 24 months is $27.9 \%$, the high rate of ineffective treatment is $57.4 \%$. In patients discharged with closure of decay cavities, the success rate is $77.5 \%$.

When comparing the results of treatment of these two groups of patients, a significant and significant difference is observed: successful treatment within 24 months is, respectively, 27.9 and $77.5 \%(p<0.05)$, the frequency of ineffective treatment is 57.4 , and $7,7 \%(\mathrm{p}<0.05)$.

Long-term results of treatment, at 48 month, data groups of patients were as follows: clinical cure amounted rule 38.2 and $72.7 \%(\mathrm{p}<0.05)$, mortality, respectively, 36.7 and
$7.5 \%(\mathrm{p}<0.05)$, including death from TB -30.9 and 5,\% (p $<0.05)$, respectively. Progression of the tuberculosis process with subsequent chronicity and development of physical condition -35.3 and $5.0 \%(\mathrm{p}<0.05)$.

During the observation period over 4 years in the group of patients with MDR MBT discharged with the destruction of lung tissue in 32 patients (47.1\%) had a different character amplification DR: 6 (8.8\%) patients with MDR MBT moved in the forward preceding-XDR MBT and $26(38.2 \%)$ developed XDR-MBT. In the group with MDR MBT, discharged with closure of the decay cavities, DR amplification was observed in $2(5 \%, \mathrm{p}<0.05)$ patients: in one MDR MBT passed into preceding-XDR, and in one - into XDR MBT.

The length of stay of patients with MDR MBT (cohort 2012-2015) in the hospital is as follows: in the group of those discharged with destruction of lung tissue, $59.5 \%$ were in the hospital for up to 8 months, and in the group of those discharged with closure of decay cavities - $68.3 \%$ ( $p>0.05)$, in longer terms, 40.5 and $31.3 \%$, respectively ( $p>0.05$ ). The results of treatment of patients with MDR MBT, cohort 20122015, are presented in Table 3.

\begin{tabular}{|c|c|c|c|c|c|}
\hline \multirow{2}{*}{ Outcomes } & \multicolumn{2}{|c|}{ CV + } & \multicolumn{2}{|c|}{ CV- } & \multirow{2}{*}{$\mathbf{p}$} \\
\cline { 2 - 5 } & $\mathbf{n}$ & $\mathbf{\%}$ & $\mathbf{n}$ & $\mathbf{\%}$ & $<0.05$ \\
\hline Cure & 16 & 38.1 & 25 & 61.0 & $>0.05$ \\
\hline Treatment completed & 1 & 2.4 & 4 & 9.8 & $<0.05$ \\
\hline Successful treatment & 17 & 40.5 & 29 & 70.7 & $<0.05$ \\
\hline Ineffective treatment & 12 & 28.6 & 3 & 7.3 & $<0.05$ \\
\hline Death & 6 & 14.3 & 0 & 0 & $>0.05$ \\
\hline a) from tuberculosis & \multirow{2}{*}{6} & 7.1 & 4 & 9.8 & $>0.05$ \\
\hline b) from other reasons & 4 & 9.5 & 5 & 12.2 & $>0.3$ \\
\hline
\end{tabular}

Table 3: Results of treatment of patients with MDR MBT, cohort 2012 - 2015.

Note: $\mathrm{p}$ - test $\chi 2$.

As can be seen from Tables 3, treatment of patients discharged with destruction of lung tissue, was significantly lower. Successful treatment was $40.5 \%$, while in patients with closure of decay cavities - 70.7\% ( $\mathrm{p}<0.05)$, ineffective treatment was 28.6 and $7.3 \%(\mathrm{p}<0.05)$, mortality -9.8 and $21.4 \%$ ( $p<0.05$ ), respectively. In individuals discharged with closure of decay cavities, decreased treatment efficacy is associated with loss to follow-up and death from other causes. Clinical cure after 36 months was $54.7 \%$ and $73.1 \%$, respectively $(\mathrm{p}<0.05)$. With continued destruction, mortality was $21.4 \%$, of which $14.3 \%$ is death from $\mathrm{TB}$, while when destruction is eliminated $-9.8 \%$ is death only from other causes. In $16.7 \%$ of cases, the formation of FCT is observed against the absence of this fact when the decay cavities are closed.

DR amplification in the main group was observed in 12 people (28.6\%): 8 patients (19.0\%) developed precedingXDR-MBT from MDR and 4 - XDR-MBT (9.5\%). In the comparison group, amplification was observed in 3 patients (7.3\%, $\mathrm{p}<0.05)$, MDR MBT passed into pre-XDR MBT.

These data indicate a significant deterioration in DST results during reactivation of the tuberculous process. The development of XDR-MBT in almost every third patient (9.5$38.2 \%$ ) should be considered especially unfavorable. It is known that treatment for XDR-TB is much more difficult, its effectiveness decreases, and the epidemiological danger is 
much higher.

The results obtained in the main group should be regarded as unsatisfactory (low), taking into account the fact that at the initial (stationary) stage of therapy, the vast majority of bacteria stopped excreting, treatment in the intensive phase was recognized as successful, and they were transferred to the continuation phase. In addition, there were no chronic forms of the disease at the initial stages, i.e., all patients could be considered curable.

After evaluating the available data, we tried to determine predictors of the retention of decay cavities during chemotherapy. Using the analysis "Logistic regression", the prognostic value of the studied indicators was determined. Initially, logistic regression was performed on the outcome of only one independent parameter.

For the analysis, the results of clinical, laboratory, and $\mathrm{X}$-ray studies were taken. Et on were the following: Gender - female, male; Age - up to 20 years old, 20-29 years old, 30-39 years old; The clinical form of tuberculosis is focal in the decay phase, infiltrative in the decay phase cavernous; Cavity diameter - up to $2 \mathrm{~cm}, 2-4 \mathrm{~cm}, 4 \mathrm{~cm}$ and mor; Number of cavities - 1 cavity, 2 cavities or more; The nature of the destructive changes - the disintegration of the lung tissue, the formed decay cavity, the formed decay cavity; Localization of the cavity - a cavity in the 1-2 segment, a cavity in the 6th segment, a cavity in other segments; The prevalence of the process - a process within 1-2 segments, a process within more than 2 segments; Bacterial excretion - MBT +, MBT-; Drug susceptibility test results - drug susceptibility, mono - resistance, multi - resistance, MDR, XDR; Revealing - re-identified, re-treated; Risk factors - alcohol dependence syndrome (SDA), gastrointestinal tract diseases, arrived from prison, pregnancy and childbirth, diabetes mellitus, chronic nonspecific respiratory diseases, contact, more than 2 years radiographically not examined, several factors at the same time; Work - works, does not work; Terms of abacillation up to 3 months, 3-4 months, 5-6 months, 7-8 months, 9-12 months, no abacillation.

To assess the role of certain features in the closure decay cavities cohort of patients $(n=105)$ was divided into 2 groups: the 1 st - patients who on the background of chemotherapy (CT), cavity decay persisted $(n=72) ; 2$ nd - patients in whom healing of the decay cavity was observed against the background of chemotherapy $(n=$ 33).

The conducted logistic regression allowed the following variables to be included in the model: gender, clinical form of tuberculosis, number of cavities, nature of destructive changes, prevalence of tuberculous process, nature of drug resistance, identification, alcohol dependence syndrome, gastrointestinal tract diseases, diabetes mellitus, contact, presence several risk factors at the same time, the timing of abacillation.

In practical terms, it is important that the set of variables is kept to a minimum. With the help of selection procedures, it was necessary to identify the variables that are most significant in terms of the forecast. Using the method of stepwise regression, we have received a finite set of variables - predictors of conservation with destructive forms of decay cavities in patients with pulmonary TB (Table 4).

\begin{tabular}{|c|c|c|c|c|c|}
\hline \multirow{2}{*}{ Independent parameter } & \multirow{2}{*}{$\begin{array}{c}\text { Regression Equation } \\
\text { Parameter (B) }\end{array}$} & \multirow{2}{*}{$\mathbf{R}$} & \multirow{2}{*}{$\begin{array}{l}\text { The ratio } \\
\text { odds (OR) }\end{array}$} & \multicolumn{2}{|c|}{ 95\% confidence interval (CI) } \\
\hline & & & & lower bound & upper bound \\
\hline Clinical form of tuberculosis & -4.907 & 0.006 & 0.007 & 0.000 & 0.250 \\
\hline Two or more cavities & -3.682 & 0.005 & 0.025 & 0.002 & 0.326 \\
\hline $\begin{array}{l}\text { Alcohol dependence } \\
\text { syndrome }\end{array}$ & -2.169 & 0.009 & 0.114 & 0.023 & 0.580 \\
\hline Abacillation up to 3 months & 0 & 0.019 & - & - & - \\
\hline Abacillation 3-4 months & 4.115 & 0.008 & 0.016 & 0.001 & 0.348 \\
\hline Abacillation 5-6 months & 5.189 & 0.002 & 0.006 & 0.000 & 0.140 \\
\hline Absence of abacillation & 25,249 & 0009 & 0.000 & 0.000 & - \\
\hline Constant & 6.456 & 0.000 & 636,293 & - & - \\
\hline
\end{tabular}

Table 4: Predictors of preservation of decay cavities in patients with destructive pulmonary tuberculosis. Note: $\mathrm{p}$ - Wald statistic.

Thus, the following variables were included in the model: clinical form (CF) of pulmonary TB, the number of decay cavities (NC), the presence of alcohol dependence syndrome (ADS), and the time of abacillation (A). Based on the results obtained, a regression equation was constructed to predict the possible preservation of decay cavities against the background of chemotherapy: 


\section{$\mathrm{Z}=6.456-4.907 * \mathrm{CF}-3.682 * \mathrm{NC}-2.169 *$ ADS $-\mathrm{A}$,}

where: $\mathrm{CF}$ (clinical form) $=0$ for infiltrative pulmonary TB in the decay phase; $\mathrm{CF}=1$ with cavernous ;

$\mathrm{NC}$ (number of decay cavities) $=0$ in the presence of 1 decay cavity; $\mathrm{NC}=1$ in the presence of 2 cavities or more;

ADS - alcohol dependence syndrome (0 - no sign, 1 - there is a sign);

$\mathrm{A}=0$ Abacillation received within 3 months; $\mathrm{A}=4.115-3-4$ months; $\mathrm{A}=5.189$ - 5-6 months; $\mathrm{A}=25.249$ - in the absence of abacillation.
To determine the stability and efficiency of the constructed model, the probability of preserving decay cavities, an assessment of its performance was carried out on the examination sample $(n=105)$. Evaluation of the effectiveness of treatment was carried out according to the criteria generally accepted in phthisiology - the frequency of abacillation and closure of the decay cavities.

Comparison of the forecast results and the obtained data in the examination sample allowed us to calculate the sensitivity, specificity and predictive value of the model; for this, a contingency table was used (Table 5).

\begin{tabular}{|c|c|c|}
\hline \multirow{2}{*}{ Group $(\mathbf{n}=\mathbf{1 0 5}$ ) } & \multicolumn{2}{|c|}{ Exodus } \\
\cline { 2 - 3 } & cavity closed & the cavity did not close \\
\hline Cavity closes camping & 32 & $\begin{array}{c}14 \\
\text { (False positive results) }\end{array}$ \\
\hline The cavity will not close & (The true positive result) & 58 \\
( false negative result) & (True negative result) \\
\hline
\end{tabular}

Table 5: A contingency table to assess the sensitivity, specificity and predictive value of the closure model for destructive pulmonary TB.

The sensitivity of the method is $97.0 \%$, the specificity is $80.6 \%$. The predictive value of a positive result is $69.6 \%$, the predictive value of a negative result is $1.69 \%$, and the diagnostic efficiency is $88.8 \%$.

Using ROC -analysis was constructed ROC curve of $t$ and defined point of separation -1.304 . When the $Z \geq-1,304$ Blog Entries: anticipate closing-cavity disintegration under chemotherapy, if the $\mathrm{Z}<-1.304$ - the collapse of the cavity is closed.

\section{Conclusion}

The results obtained indicate a significant decrease in the effectiveness of treatment of patients discharged with the preservation of the destruction of lung tissue, even under the condition of abacillation; high risk of developing FCT (up to $35.3 \%$ ), amplification of MBT DR, increased mortality from TB (33.8\%). In assessing the effectiveness of treatment of patients with destructive pulmonary TB, it is necessary to take into account the closure rate of decay cavities, not being limited to one criterion of abacillation. It is necessary to resolve the issue of using modern surgical methods and / or collapse-forming techniques at earlier stages of treatment, up to 6 months, the feasibility of developing and implementing methods for predicting the likelihood of preserving decay cavities. The results of predicting the possibility of preserving decay cavities against the background of chemotherapy allow a practitioner to use the main predictors of closure of decay cavities in each specific case. This will allow for a short time to predict the possibility of preserving decay cavities with a sensitivity of $97 \%$, specificity $-80.6 \%$ and to determine the tactics of therapy for such patients, the expediency of the timely use of surgical or collapsotherapy methods in the complex treatment of destructive pulmonary TB.

Conflict of Interest: The authors declare that they have no conflicts of interest.

\section{References}

1. Balasanyants GS (2017) Experience of using bedaquiline in the complex treatment of patients with tuberculosis in combination with HIV Infection. Tub and lung disease 95(9): 49-54.

2. Krivonos PS (2018) Prevalence of pulmonary tuberculosis with multidrug-resistant mycobacterium tuberculosis in modern conditions. Modern trends in the development of respiratory medicine and phthisiology: materials of the First Congress of phthisiatricians and pulmonologists of the Republic of Belarus, Minsk, May 17-18, 2018 / State Institution Republican Scientific Practical Center "Pulmonology and phthisiology; editorial boar: GL Gurevich [and others]. - Minsk, pp: 8487.

3. Skornyakov SN (2015) Surgery of destructive drugresistant pulmonary tuberculosis. Tub and lung disease 3: $15-21$.

4. Suyundikov IK (2017) Tuberculosis incidence in Russia as a social problem. Bul honey and internet conferences 


\section{Journal of Infectious Diseases \& Travel Medicine}

6: 928-929.

5. Kawatsu L, Uchimura K, Izumi K, Ohkado A, Yoshiyama $\mathrm{T}$ (2018) Treatment outcome of multidrug-resistant tuberculosis in Japan - the first cross-sectional study of Japan tuberculosis surveillance data. BMC Infect Dis 31: 445.

6. Sterlikov SA, Testov VV, Vasilyeva IA (2015) Results of treatment of patients with multiple and extensively drug-resistant pathogens registered in 2012. In: The Russian Federation and in the world. Tub and lung disease 3: 22-27.

7. Gaipov RG (2017) Palliative surgery in the treatment of destructive pulmonary tuberculosis in patients with multidrug-resistant. Science, new technologies and innovations in Kyrgyzstan 8: 86-88.
8. Igembaeva KS (2014) Social status and outcomes of hospital treatment of patients with tuberculosis. Science and Health 2: 84-85.

9. Porkulevich NI (2015) Factors contributing to the chronicization of tuberculosis in adults on the example of the Omsk region. Young Russia: advanced technologies in industry 3: 236-241.

10. Seltsovsky PP, Plieva SL, Svistunova AS (2015) Risk factors for the development of early relapses of respiratory tuberculosis. Tub and socially significant diseases 3: 4-10.

11. Lange C, Chesov D, Heyckendorf J (2018) Clofazimine for the treatment of multidrug-resistant tuberculosis. Clin Microbiol Infect 25(2): 128-130. 